\title{
Rôle dynamique de CSF1R nucléaire dans la lignée monocytaire
}

Laura Bencheikh ${ }^{1,2}$, Aygun Imanci ${ }^{1,2}$, Alexia Plocque ${ }^{1,2}$, Éric Solary ${ }^{1-3}$, Nathalie Droin ${ }^{1,2,4}$

> Typiquement, les récepteurs à activité tyrosine kinase (RTK) sont des glycoprotéines transmembranaires qui, en réponse à la fixation d'un ligand, induisent des signaux intracellulaires stimulant la prolifération, la différenciation, et d'autres fonctions cellulaires. Chez l'homme, il existe 58 RTK classés en 20 sous-familles [1]. Ces récepteurs sont le plus souvent constitués d'une partie extracellulaire sur laquelle se fixe un ligand, d'un domaine juxta-membranaire ayant un rôle régulateur, d'un domaine portant l'activité kinase, et d'une région C-terminale. La fixation du ligand induit la dimérisation du récepteur et active sa fonction kinase par auto- ou trans-phosphorylation du domaine cytoplasmique [2]. Cette phosphorylation et le changement de conformation permettent le recrutement de protéines (kinases, phosphatases, protéines adaptatrices, etc.) impliquées dans la signalisation intracellulaire. Pour interrompre cette activité enzymatique, la cellule internalise le récepteur et le dégrade, généralement dans ses lysosomes, ou le recycle à la membrane plasmique après l'avoir inactivé.

Plusieurs de ces récepteurs sont également localisés dans le noyau (en anglais, membrane receptor in nucleus, ou MRIN). Des MRIN ont en effet été identifiés dans 12 des 20 sous-familles de RTK. Ils sont présents dans le noyau sous une forme entière (holorécepteur) ou sous la forme de molécule tronquée. Ils participent à la régulation transcriptionnelle et à la réparation de I'ADN. CSFIR (colony stimulating factor 1 receptor) ou M-CSFR (macrophage colony-stimulating factor receptor) sont des MRIN. CSFIR joue un rôle essentiel dans le lignage monocytaire et macrophagique. Ses ligands sont le CSFl et l'interleukine-34 (IL-34). II est anormalement exprimé par les cellules de nombreuses lignées cancéreuses d'origine épithéliale. C'est un RTK de classe III qui comporte deux domaines à activité kinase séparés par un domaine $\mathrm{KI}$ (kinase insert). Cette structure est caractéristique d'une famille dans laquelle on trouve aussi PDGF-R ( $p l a-$ telet-derived growth factor receptor), KIT/SCFR (mast/stem cell growth factor receptor), et FLT3 (Fms-like tyrosine kinase 3). Seuls PDGF-R et CSFIR ont été détectés dans le noyau. CSFIR a d'abord été observé dans le noyau de macrophages murins et de cellules cancéreuses humaines. Cette localisation nucléaire a été attribuée à l'isoforme pl10 $\delta$ de la phosphoinositide 3-kinase (PI3K) et à la GTPase Rab5. Dans le noyau, CSFlR s'est montré capable de phosphoryler les protéines p27 et Akt (ou protéine kinase B) [3]. Dans des cellules cancéreuses humaines, CSFIR a aussi été détecté sur le promoteur des gènes CCNDI (cyclin D1), MYC, JUN et CSFI [4], suggérant un rôle dans le contrôle transcriptionnel de la prolifération cellulaire.

$\varepsilon$ n combinant plusieurs formes de microscopie, nous avons aussi détecté CSFIR dans le noyau des monocytes humains circulants et dans celui des macrophages issus de la différenciation de ces monocytes en réponse à un
IInserm U1170, Gustave Roussy,

94805 Villejuif, France.

2Université Paris-Sud, faculté de médecine, 94270 Le Kremlin-Bicêtre, France.

${ }^{3}$ Département d'hématologie, Gustave

Roussy, 94805 Villejuif, France.

${ }^{4}$ Inserm US23, CNRS UMS 3655, AMMICa, plateforme génomique, Gustave Roussy, 94805 Villejuif, France.

nathalie.droin@gustaveroussy.fr

ligand de CSFlR. C'est l'holorécepteur qui se retrouve dans le noyau de ces cellules et l'utilisation d'un ligand fluorescent a montré que le récepteur migre dans le noyau avec son ligand. Cette translocation nucléaire est bloquée par deux petites molécules inhibitrices de la fonction kinase de CSFIR, BLZ945 et GW2580. Par immunoprécipitation de la chromatine suivie de séquençage (ChIPseq), nous avons détecté 4980 «pics » CSFIR sur l'ADN des monocytes de sujets sains. Certains de ces pics étaient localisés sur le promoteur du gène PU.1/SPII (purine-rich nucleic acid-binding protein 1/ SFFV provirus integration site-1), codant un facteur de transcription de la différenciation myélo-monocytaire, et sur le site d'initiation de la transcription de CSF2RB, codant une des chaînes du récepteur du GM-CSF (granulocyte monocyte-(SF). Plus globalement, les motifs de l'ADN où les pics CSFIR ont été détectés correspondent aux sites de fixation des facteurs de transcription $\varepsilon G R$ (early growth response). Des ChIP-seq ciblant EGRI ont montré une co-localisation des pics CSFlR et EGRl, le plus souvent dans les zones intergéniques, avec la marque H3K4mel (histone $\mathrm{H} 3$ mono-methylation at lysine 4). Nous avons renouvelé ces expériences après avoir exposé les monocytes à CSFl, ligand de CSFIR, et avons alors observé une relocalisation de ce récepteur aux sites d'initiation de la transcription dès la sixième heure, un effet que nous avons également observé après 3 jours d'exposition à CSFl (Figure 1). CSFIR est alors co-localisé avec la marque 


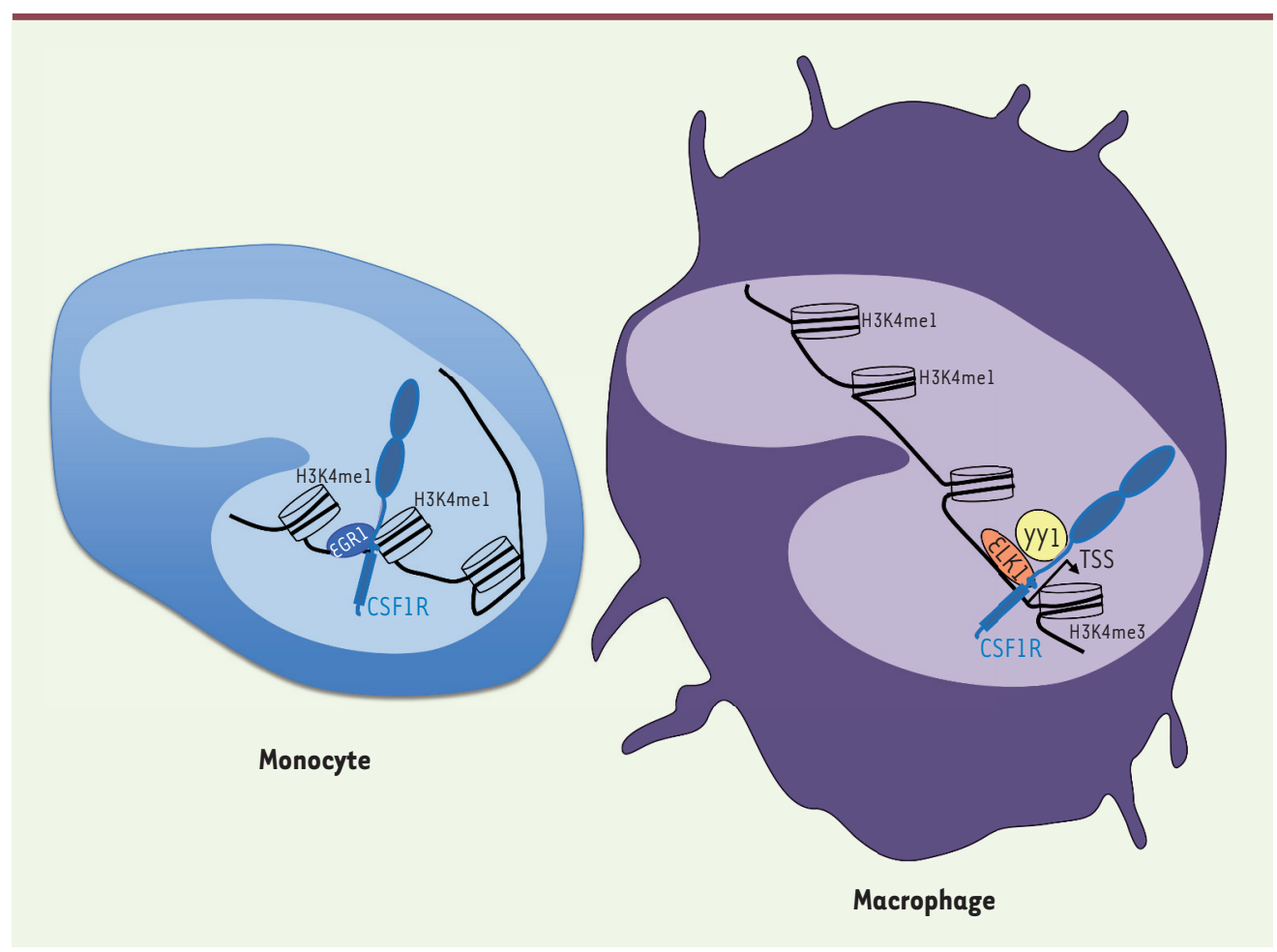

Figure 1. Les interactions de CSFIR avec la chromatine diffèrent dans les monocytes et dans les macrophages issus de la différenciation de ces monocytes. Dans les monocytes, CSFIR interagit avec $\varepsilon G R l$, et est colocalisé avec H3K4mel dans les régions intergéniques. Dans les macrophages, CSFIR interagit avec ELKI ou yyl, et est co-localisé avec H3K4me3 aux sites initiateurs de la transcription (transcription start sites, ou TSS).

H3K4me3 (histone H3 tri-methylation at lysine 4). Les motifs de I'ADN où les pics CSFIR sont détectés correspondent aux sites de fixation des facteurs de transcription $\varepsilon 2 F 4$ ( $\varepsilon 2 F$ transcription factor 4), ELK (ETS transcription factor), et yyl (ying yang 1). Des expériences de co-immunoprécipitation ont confirmé l'interaction de CSFIR avec ELKl et Yyl. La leucémie myélo-monocytaire chronique est une hémopathie myéloïde clonale associée au vieillissement dans laquelle des monocytes s'accumulent dans la moelle osseuse et le sang. L'expression de CSFIR peut être diminuée dans les cellules du clone leucémique. Quand il est exprimé, CSFlR est encore détecté sur la chromatine, mais dans des régions distinctes de celles identifiées dans les monocytes de sujets sains [5].

La fonction de CSFIR ne se limite donc pas à l'induction de signaux intracellulaires à partir de la membrane plasmique, mais comporte également des rôles nucléaires. Cette découverte pourrait éclairer plusieurs aspects de la différenciation myélo-monocytaire normale et pathologique. Pendant des années, l'engagement d'une cellule souche hématopoïétique dans une voie de différenciation a été attribué aux variations stochastiques de l'équilibre entre facteurs de transcription. En 2013, l'équipe dirigée par Michael Sieweke a montré que CSFl induisait l'expression du gène $P U .1$, un puissant régulateur de la différenciation myéloïde, dans des cellules souches hématopoïétiques murines, suggérant un effet instructeur de facteurs extrinsèques sur leur différenciation [6]. La présence de CSFIR dans le noyau des cellules souches et progénitrices hématopoïétiques suggère que cet effet instructeur pourrait s'exercer également sur I'ADN. La forme nucléaire de CSFIR pourrait aussi contribuer à l'hétérogénéité des monocytes circulants. Depuis une dizaine d'années, une nomenclature internationale distingue trois populations de monocytes selon le taux d'expression des gènes CD14 et CD16 (cluster of differentiation 14 and 16) : les monocytes classiques $\left(\mathrm{CD} 14^{++} / \mathrm{CD} 16^{-}\right)$, intermédiaires $\left(\mathrm{CDI}_{4}^{++} / \mathrm{CDI}^{++}\right)$, et non-classiques
$\left(\mathrm{CD} 14^{-} / \mathrm{CDI}^{++}\right)$constituent respectivement environ $85 \%, 5 \%$, et $10 \%$ des monocytes circulants. Les mécanismes à l'origine de cette hétérogénéité restent controversés, mais une partie au moins des monocytes intermédiaires et nonclassiques dérivent des monocytes classiques [7]. Les mécanismes moléculaires de cette conversion, encore imparfaitement compris, impliquent notamment un axe miR150/TET3 (microRNA150 / methylcytosine dioxygenase 3), dérégulé par un mécanisme épigénétique dans la leucémie myélo-monocytaire chronique [8]. CSFlR est aussi impliqué dans cette conversion puisque, dans la leucodystrophie héréditaire diffuse (hereditary diffuse leukodystrophy with axonal spheroids), une microgliopathie rare due à des mutations constitutives inactivatrices du domaine tyrosine kinase de CSFIR, on observe une diminution du nombre de monocytes intermédiaires et non-classiques [9]. En outre, un anticorps bloquant CSFIR, utilisé dans le traitement de la polyarthrite rhumatoïde, entraîne une déplétion des monocytes non-classiques $[10]$. II reste à 
déterminer si les fonctions nucléaires de CSFlR jouent un rôle dans la conversion d'un monocyte classique en monocyte non-classique, et si la perturbation de ces fonctions observée dans la leucémie myélo-monocytaire chronique contribue également à la répartition anormale des populations monocytaires caractéristique de cette maladie. $\diamond$

Dynamic role of nuclear CSFIR in the monocytic lineage

\section{LIENS D'INTÉRÊT}

Les auteurs déclarent n'avoir aucun lien d'intérêt concernant les données publiées dans cet article.

\section{RÉFÉRENCES}

1. Blume-Jensen $P$, Hunter T. Oncogenic kinase signalling. Nature 2001 ; 411 : 355-65.

2. Lemmon MA, Schlessinger J. Cell signaling by receptor tyrosine kinases. Cell $2010 ; 141$ : 1117-34.

3. Zwaenepoel 0, Tzenaki N, Vergetaki A, et al. Functional CSF-1 receptors are located at the nuclear envelope and activated via the $\mathrm{pl} 10 \delta$ isoform of PI 3-kinase. FASEB J 2012 ; 26 : 691-706.

4. Barbetti V, Morandi A, Tusa I, et al. Chromatinassociated CSF-1R binds to the promoter of proliferation-related genes in breast cancer cells. Oncogene 2014 ; 33 : 4359-64.

5. Bencheikh L, Diop MK, Rivière J, et al. Dynamic gene regulation by nuclear colony-stimulating factor 1 receptor in human monocytes and macrophages. Nat Commun 2019; $10: 1935$

6. Mossadegh-Keller N, Sarrazin S, Kandalla PK, et al. M-CSF instructs myeloid lineage fate in single haematopoietic stem cells. Nature 2013 ; 497 : 239-43.

7. Patel AA, Zhang $Y$, Fullerton JN, et al. The fate and lifespan of human monocyte subsets in steady state and systemic inflammation. J Exp Med 2017 ; 214 : 1913-23.

8. Selimoglu-Buet D, Rivière J, Ghamlouch H, et al. A miR-150/TET3 pathway regulates the generation of mouse and human non-classical monocyte subset. Nat Commun 2018 ; 9 : 5455.

9. Hofer TP, Zawada AM, Frankenberger M, et al. Slandefined subsets of CD16-positive monocytes: impact of granulomatous inflammation and M-CSF receptor mutation. Blood 2015; 126 : 2601-10.

10. Korkosz M, Bukowska-Strakova K, Sadis S, et al. Monoclonal antibodies against macrophage colonystimulating factor diminish the number of circulating intermediate and nonclassical $\left(\mathrm{CD} 14^{++} \mathrm{CD}_{16}{ }^{+}\right.$) $\left.\mathrm{CD} 4^{+} \mathrm{CD} 6^{++}\right)$monocytes in rheumatoid arthritis patient. Blood $2012 ; 119$ : 5329-30.
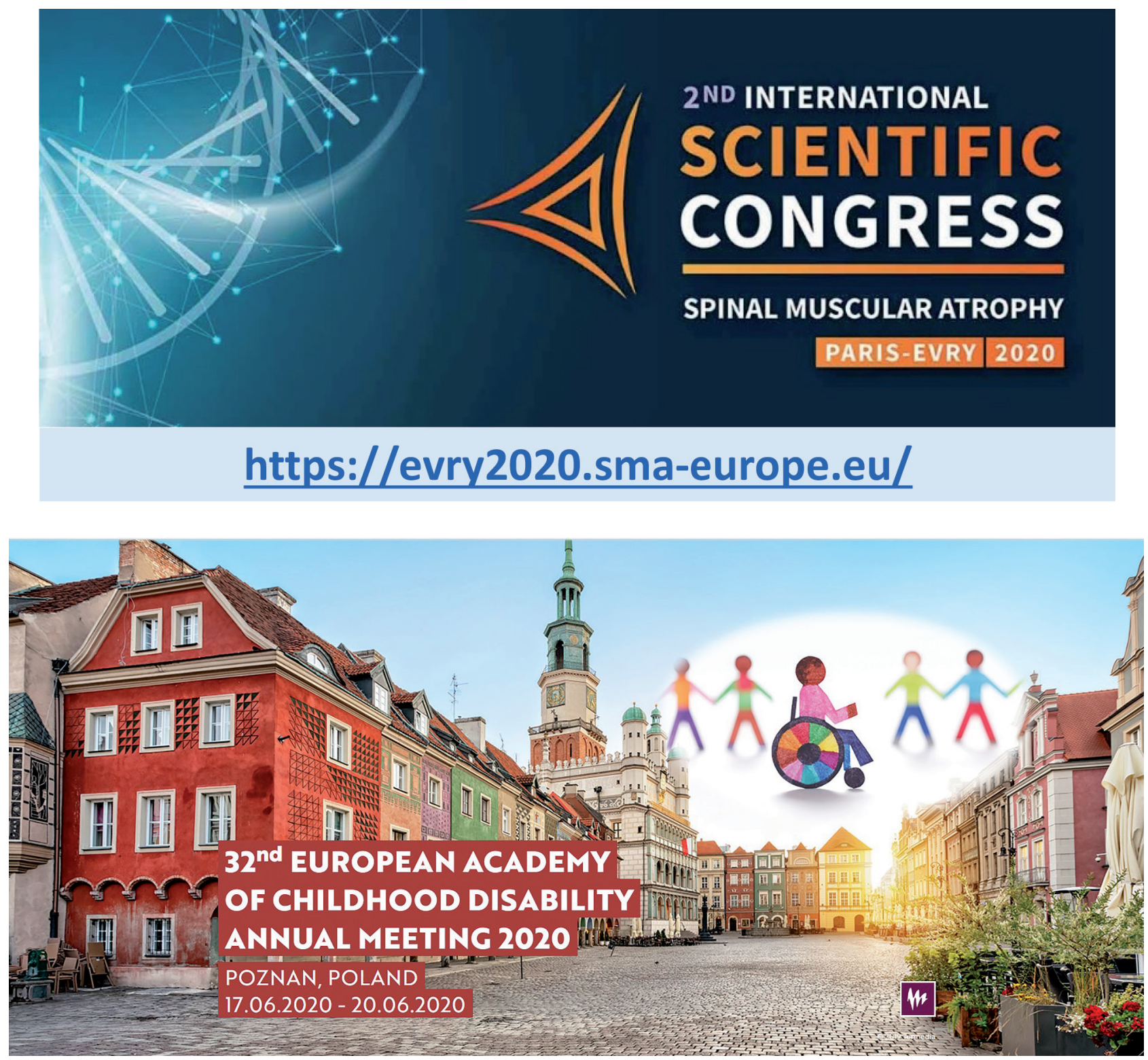